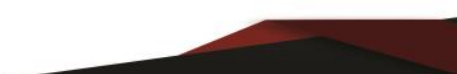

Comment

\title{
O Trabalho Médico nas Unidades de Terapia Intensiva (UTI's) e a propensão ao desenvolvimento de Distúrbios Psicológicos
}

\author{
Pedro Henrique de Holanda Júnior ${ }^{1}$; Francisca Silva de Alencar ${ }^{2}$; Juliane de Oliveira Costa Nobre ${ }^{3}$
}

Resumo: Este artigo buscou refletir a saúde do médico que atua em unidade de terapia intensiva, por ser este um ambiente de trabalho onde o estresse parece decorrer, principalmente, por tratar-se de um ambiente fechado, com uma carga laboral mais intensa, demandando demasiada atenção e concentração do profissional. O risco de morte eminente e a proximidade e convívio exaustivo com situações de maior sofrimento e dor, agravam a possibilidade de doenças emocionais. A literatura tem destacado que, o incentivo a manutenção de uma qualidade de vida por esses profissionais, configura uma forma de prevenir o desencadeamento de enfermidades físicas e mentais.

Palavras-chave: Saúde do trabalhador, estresse laboral, Medicina e trabalho.

\section{The Medical Work in Intensive Care Units (ICUs) and the propensity to develop Psychological Disorders}

abstract: This article aims to reflect the health of the doctor who works in an intensive care unit, because this is a work environment where stress seems to be mainly due to the fact that it is a closed environment, with a more intense workload, demanding too much attention and professional concentration. The risk of eminent death and the proximity and exhaustive conviviality with situations of greater suffering and pain, exacerbate the possibility of emotional illness. The literature has emphasized that the incentive to maintain a quality of life by these professionals is a way to prevent the onset of physical and mental illnesses.

Keywords: Worker's health, Work stress, Medicine and work.

\footnotetext{
${ }^{1}$ Enfermeiro Assistencialista no Hospital Universitário Onofre Lopes. Especializando em Enfermagem do Trabalho pela Faculdades Integradas de Patos, PB.

${ }^{2}$ Mestranda em Ciências da Saúde pela Faculdade de Medicina do ABC - FMABC, Santo André, São Paulo. Contato: silvia.ramo@hotmail.com;

${ }^{3}$ Doutora em Ciências da Saúde pela Faculdade de Ciências Médicas da Santa Casa de São Paulo - FCMSCSP.
} 


\section{Introdução}

As unidades de terapia intensiva (UTI's) consistem em local destinado a pacientes com quadro clínico grave ou com altos riscos de morte, que carecem de uma assistência médica específica e qualificada, de equipamentos tecnológicos adequados, bem como de se manter em observação e monitoração constante. Com isso, é normal que seja local de forte estresse, tanto familiar, quanto do paciente e do médico que acompanha o caso.

Este estudo tem como objetivo, discutir, à luz da literatura especializada, sobres aspectos relacionados ao trabalho do médico intensivista, mais precisamente sobre sua saúde laboral. Foram utilizados os trabalhos de Kovács (2010); Garcia (2010); Fiorotti et al (2010); Benevides-Pereira (2009); Lopez et al (2006); Maslach et al, 2001). Meleiro (1998); Leung e Becker (1992), dentre outros, condensando seus principais resultados.

O estresse tem revelado um viés altamente negativo para a equipe médica atuante, o que tem ocasionado enfermidades físicas e mentais no corpo profissional do ambiente intensivista. Assim, além do cansaço físico e da predisposição para ser contagiado com doenças; tem-se verificado a presença de enfermidades psicológicas nesses profissionais, dentre as quais encontramos a recorrência de Transtorno Mental Comum (TMC) e da Síndrome de Burnot (BENEVIDES-PEREIRA, 2009).

O estresse do ambiente de medicina intensivista decorre, principalmente, do fato de ser um ambiente fechado, com elevado ritmo de trabalho e excesso de carga laboral, que demanda demasiadamente do médico tanto profissionalmente, quanto emocionalmente; em razão da proximidade e convívio constante com situações de sofrimento e risco morte (MELEIRO, 1998).

Observe-se e, como esperado, um dos primeiros sintomas desenvolvido pelo médico, é a exaustão, devido a presença de uma sobrecarga física e psicológica a qual ele é submetido. Esta é uma queixa comum entre intensivistas. Isso interfere diretamente na sua vida pessoal e social, tornando-o um indivíduo isolado e desgastado, podendo inclusive, passar a adotar uma postura impessoal e fria no ambiente laboral, seja com os pacientes ou com os colegas de trabalho. Isto porque os médicos tendem a utilizar isso inconscientemente como ferramenta psíquica de defesa, incorporando, consequentemente ao seu comportamento no ambiente de 
trabalho; o que revela a possibilidade da presença de um quadro clínico de enfermidade psíquica.

A privação do sono, a presença constante de situações ansiogênicas, o excesso de trabalho, o estresse do ambiente ante as demandas de urgência, a proximidade com a constante possibilidade de risco de morte, ou seja, as características comuns de um trabalho em UTI têm sido apontados como principais fatores de desencadeamento de enfermidades mentais (KOVÁCS, 2010).

O Estresse emocional presente no ambiente intensivista, compromete a saúde do médico, bem como põe em risco sua qualidade de vida, podendo, ainda, repercutir negativamente na sua atuação como profissional. Corroborando com esse entendimento, estudos demonstram que os índices de suicídio são maiores entre os profissionais da medicina, do que nas outras profissões (MELEIRO, 1998).

Assim, os diversos vínculos laborais e o excesso de carga horária de trabalho, acompanhado com uma remuneração considerada baixa para o quantitativo e tipo de trabalho prestado, juntamente com o estresse mental ao qual são submetidos em uma UTI, têm se revelado ponto negativo para a saúde mental do profissional da medicina. Principalmente porque costumam trabalhar em jornadas longas de 36 horas ou mais, quase sem conseguir dormir, o que acaba interferindo negativamente em seus estados de humor, na sua vida pessoal e profissional.

Esse ambiente de excesso de trabalho e intenso estresse emocional, tem se revelado um facilitador para o desenvolvimento de enfermidades psíquicas, como já mencionado. Com base nisso, o presente artigo estudará a Síndrome de Burnout, que está intimamente relacionada com o sofrimento do profissional no ambiente em que trabalha, que vem acompanhado de uma perda de motivação e insatisfação pessoal e profissional; e o Transtorno Mental Comum, caracterizado pela presença de sintomas que revelam depressão, ansiedade, ausência de sono, dificuldade de concentração, irritação, cansaço extremo e outras reclamações, sendo um tipo de quadro clínico que envolve basicamente ansiedade, depressão e sintomas somáticos. 


\section{Fatores que interferem negativamente na saúde mental do médico intensivista}

O ambiente e condições do trabalho interferem consideravelmente no íntimo e pessoal de um trabalhador. Não diferente ocorre com os profissionais de medicina das UTI's, que consiste em ambiente carregado de variabilidades, inquietação, insalubridade, dor, dentre outros; todos fontes de estresse e cansaço, o que contribui indubitavelmente para a possibilidade de lesões físicas e desencadeamento de distúrbios mentais ou comportamentais.

O médicos plantonistas das UTI's enfrentam constantemente situações graves no trato com pacientes, momentos de pressão pelo alto quantitativo de pessoas para atender e prestar assistência e um excesso de carga laboral, que interferem na sua vida como um todo, privandoo de um sono de qualidade, de uma alimentação adequada, do convívio social e familiar, de momentos de lazer, dentre outros (BATES, HINTON e WOOD, 1973; LEUNG e BECKER, 1992; ASKEN e RAHAM, 1983).

Trata-se de ambiente com grande exposição a riscos químicos e biológicos, em razão da variedade de enfermidades presentes; físicos e ergonômicos, devido ao quantitativo de horas sequencialmente trabalhadas; bem como psíquicos, em razão das situações de estresse as quais os médicos são submetidos; o que pode ocasionar o desequilíbrio do bem-estar psicossocial e consequente desencadeamento de problemas de saúde nesses profissionais.

Consiste em um quadro clínico que se instaura, muitas vezes, de forma silenciosa e imperceptível, dando origem a transtornos depressivos, síndromes metabólicas, síndrome de Burnot, transtornos mentais, dentre outros; momento em que é perceptível a presença de sintomas de estresse, frustração profissional, dificuldade concentração e distúrbios cognitivos.

O estresse, sem dúvidas, trata-se de elemento potencializados dor sintomas. Além disso, o quantitativo horas de plantões em conjunto com a insuficiência do valor pago pelo tipo e quantitativo do serviço prestado, são fatores de risco de instauração de quadros clínicos psicológicos. Ademais, há fatores como exigência da manutenção de um ritmo de atendimento e alcance de metas; uma infraestrutura insuficiente; equipamentos inadequados; e presença de um ambiente inadequado e desconfortável; que se apresentam como agravantes dos desgastes físicos e mentais pelos médicos.

Por fim, é importante comentar que esses momentos de preocupação, tensão, medo e estresse, que os médicos plantonista de unidades intensivistas passam constantemente passam, chama atenção para serem essas as principais causas do desenvolvimento de enfermidades 
psicológicas nesses profissionais. Além disso, notou-se que as UTI's são ambientes que carregam uma tensão tão grande, que os profissionais tendem a esquecer deles mesmos em prol do cuidado com o paciente, deixando, inclusive de cuidar de sua própria saúde, o que facilita o surgimento dos efeitos maléficos dessa rotina em seu organismo (GARCIA, 2010).

\section{O desenvolvimento do Transtorno Mental Comum (TMC) em profissionais da medicina plantonistas de UTI's}

Em 1970, a definição de Transtornos Mental Comum foi elaborada pelo médico psiquiatra David Goldberg em conjunto com o professor Peter Huxley (1992, p. 194), para se referir a um conjunto de sintomas, como insônia, cansaço extremo, lapsos de memória, irritação, estresse, desconcentração e outras queixas, relacionadas ao desenvolvimento de uma enfermidade mental ocorrida com recorrência no âmbito da atenção primária salutar, sendo este o local em que se encontra o mais quantitativo pessoas com transtornos não psicóticos em comparação com os casos psiquiátricos.

Esses transtornos, apesar de não estar presente na doutrina nosológica como um caso clínico específica, possuem íntima correlação com transtornos mentais de ansiedade e depressão psicótica assintomática, configurando um fenômenos psicopatológico (MARI; JORGE, 1997). Inclusive, conforme informa a Organização Mundial de Saúde (OMS), os transtornos depressivos consistem na quarta causa incapacitante em nível global (LOPEZ ET AL, 2006), isto porque apesar de inicialmente não aparentarem serem graves, dão causa a um quadro situacional de intenso sofrimento, o que, posteriormente, pode dar ensejo a incapacidade e consequente impossibilidade laboral.

No meio hospitalar, o ambiente de UTI's consiste em um dos locais mais estressantes para os profissionais da saúde, sendo o local de maior ocorrência do transtorno, principalmente em médicos que desenvolvem uma habilidade técnica médica específica, isto porque precisam ter um controle emocional muito grande, além de autoconfiança, para que seja capaz de direcionar a equipe cirúrgica para a melhor forma de solucionar o problema de maneira mais rápida possível.

O predomínio do TMC entre os médicos, está relacionado com as particularidades dessa profissão diante das responsabilidades com o paciente, já que laboram com o objetivo de salvar 
vidas. Além disso, apesar das demais profissões. A gravidade dessa enfermidade no meio médico por ser confirmada por esta ser causa do maior quantitativo de suicídio nos médicos, quando em comparação com as demais profissões também acometidas pela enfermidade (MALEIRO, 1998).

Indubitavelmente, diversos são os fatores estressantes que decorrem do vínculo médicopaciente, envolvendo o medo de errar, medo de adquirir as doenças, privação do sono, cansaço excessivo, isolamento sócia, plantão diversos lugares, condições de trabalho, ter que comunicar uma doença grave ou uma morte, dentre outros, não incluindo transtornos psicóticos, dependência química ou transtornos de personalidade. No entanto, estudos apontam que o desenvolvimento dos transtornos é mais recorrente nos médicos recém-formados, diminuindo a incidência com o passar dos anos, provavelmente porque acabam se acostumando e se conformando com a rotina estressante e suas adversidades.

O TMC pode ocasionar e favorecer o desenvolvimento de transtornos mais sérios, que envolvam o uso de substâncias psicoativas, em razão do médico sentir necessidade de utilizar medicamentos para mitigar ou tentar diminuir os sintomas sentidos, bem como o consumo de bebidas alcoólicas e substâncias entorpecentes. Pode se manifestar na forma de ansiedade, quando mais leve, ou como depressão. Enquanto ansiedade, se revela como uma preocupação excessiva, nervosismo, tensão, dores musculares, insônia, estado ansioso; quando no extremo da depressão, envolve sintomas de cansaço, perda de libido, falta de fome, autocrítica destrutiva, variações de humor, falta de concentração, insônia e lentidão (SPRICIGO, 2010).

Segundo estudos, a pessoa é diagnosticada com a enfermidade apresenta alguns sintomas que comportam uma abordagem múltipla, pois envolve sintomas de várias categorias, onde se entende que o termo inicial da doença está na fase atuação profissional, podendo também ter início durante a graduação, isto porque algumas atividade laborais tem o condão de facilitar a instauração ou agravamento do transtorno ((BENEVIDES-PEREIRA, 2009; SPRICIGO, 2010; SOBRINHO et al, 2010).

A estreita relação entre a formação do profissional de medicina e os sintomas do TMC pode ser verificada em várias etapas, desde o vestibular, que consiste na seleção mais concorrida e difícil das faculdades, gerando uma pressão no estudante além do suportável em um ambiente de extrema competitividade, causando tensão e desequilíbrio emocional antes mesmo do ingresso no meio acadêmico superior. Já na graduação, o acadêmico é submetido a um excesso 
de conteúdo de estudo e uma carga horária integral, o que limita as relações socais e lazer. Segundo Fiorotti et al (2010, p.18):

\begin{abstract}
"Alguns estudos conduzidos na área da saúde mental têm sido focados em estudantes da área da saúde, principalmente do curso de Medicina, que expõe os estudantes a fontes de tensão desde o processo de admissão até o final da graduação, com a entrada no mercado de trabalho e em programas de residência médica".
\end{abstract}

De qualquer forma, em meio as diversas possibilidade de causas do transtorno, as situações que despertam o sentimento de medo, seja medo da morte, de cometer erros ou medo do contagio de doenças lideram o placar, o que ocorre com frequência nas UTI's. Além disso, excesso de autocobrança em busca da perfeição e a competitividade, acabam gerando uma tensão desproporcional e, quando não alcançam o que objetivavam, acabam desenvolvendo problemas de autoestima relacionado com sentimento de inferioridade (BENEVIDESPEREIRA, 2009).

O TMC transforma o profissional, deixando-o psicologicamente enfermo, o que interfere em sua desenvoltura laboral, na sua concentração, ou seja, torna o indivíduo desequilibrado emocionalmente, o que pode prejudicar o médico, não só no desenvolvimento de suas atividades, como também na sua interação e comunicação com o mundo ao seu redor.

Estudos envolvendo o transtorno atestaram a facilidade de seu desenvolvimento em diversas profissões, dentre as quais encontram-se os médicos. Ainda, foi atestado a prevalência da enfermidade, dentre outros locais, nas UTI's, principalmente quando pediátrica (PITTA, 1994); e de emergências em geral, como locais mais propício ao desenvolvimento de Transtorno Mental Comum nos médicos, em decorrência, principalmente, do estresse diário e das más condições de trabalho aos quais, em regra, são submetidos.

Nesse meio de UTI's, o profissional de saúde enfrenta constantemente momentos de tensão que podem colaborar para seu desenvolvimento e aperfeiçoamento enquanto médico, ou dar causa a distúrbios mentais. Com isso, resta clara a importância do estudo do TCM em médicos, observando quais os transtornos mentais mais comumente desenvolvidos e suas variáveis sociodemográficas, bem como a correlação dessas enfermidades com formação profissional dos médicos. 


\section{A correlação entre o trabalho médico intensivista e a Síndrome de Burnout}

A Síndrome de Burnout consiste em momento de esgotamento do profissional, decorrente da exposição excessiva a problemas interpessoais crônicos no ambiente laboral, podendo dá-se em três formas: (i) exaustão emocional, caracterizada pela sensação de esgotamento pelo indivíduo; (ii) despersonalização do profissional, que diz respeito ao desenvolvimento de uma conduta pessoal fria, negativa e insensível, um tanto desumanizada e carregada de hostilidade, intolerância e impessoalidade; e a (iii) ineficácia, que consiste no médico não se sentir realizado profissionalmente, isto porque os portadores da síndrome, em regra, costumam achar que não conseguem atingir seus objetivos profissionais.

No meio médico, acomete os profissionais que trabalham com pacientes em situação de risco ou que demandem extrema responsabilidade (MASLACH et al, 2001). Ademais, conforme apontam estudos, a maioria dos plantonistas de UTI's reclamam dos ruídos excessivos, bem como na constante possibilidade de piora dos pacientes, como as duas principais causas do estresse emocional causado nesse ambiente (SOBRINHO et al, 2010).

O perfil dos médicos plantonistas, em regra, são jovens com menos de dez anos de formados, que se comprometem com uma carga horária excessiva de trabalho, principalmente em regime de plantão, que, normalmente, sequer possuem a residência médica específica para a atuação em UTI. Comente-se, ainda, que em um comparativo com o perfil de médicos operantes em outros países, a média de idade e de anos de formação também é inferior (SOBRINHO et al, 2010).

Nesse ambiente, verificou-se que o desenvolvimento da síndrome tem grande incidência nas três dimensões da enfermidade (emocional, profissional e ineficácia), podendo apresentar variações dependendo da localidade, mas sempre com maior incidência no meio médico intensivista do que nas outras especialidades. No entanto, a dimensão mais fortemente afetada é da exaustão emocional, por ser a que primeiramente é atingida em razão das severas exigência que o trabalho demanda em uma UTI.

Assim, uma vez estafados nas referidas dimensões, os médicos passam a sentir cansaço extremo, tando físico quanto emocional, dificultando, inclusive, que eles relaxem nas horas vagas, momento em que o profissional passa a apresentar mais claramente aspectos da síndrome de Burnout. Presentes os sintomas, físicos e mentais, o médico tende a desenvolver a 
despersonalização, momento em que passa a portar-se de forma fria e negativa, o que, por vezes, implica no uso de tratamentos depreciativos com os demais trabalhadores do local; podendo, ainda, apresentar um comportamento cínico e irônico com os pacientes (THOMAS, 2004).

A sequência do quadro instaurado com a despersonalização, é a ineficácia. O profissional da medicina para a se ver como insuficiente e ineficiente o trabalho desenvolvido, o que diminui sua autoconfiança e desenvolve uma sensação de fracasso, o que interfere diretamente na sua percepção de realização profissional. A maioria da doutrina entende que a ineficácia consiste na última fase da instauração da síndrome, sendo a reação mais trágica causada pelas exigências do trabalho intensivista.

Thomas (2004), ao estudar a enfermidade, atestou fortes indícios com a facilidade de desenvolver a síndrome com a dificuldade de cuidar de pacientes, seja pela gravidade do quadro clínico ou pela insuficiência de recursos, e a depressão. Corroborando, ainda, que a síndrome é influenciada tanto por características pessoais do indivíduo, tanto pelas demandas excessivas do trabalho, sendo esta última a mais recorrente.

Sobrinho et al (2010), verificaram que a enfermidade é mais comumente desenvolvida por profissionais que não possuem residência médica na área de Medicina Intensivista, bem como naqueles que se disseram ativos fisicamente ou possuidores de algum tipo de hobby. A primeira situação é a mais importante, isto porque a parcela que apresentou Burnout, era composta, em sua maioria por médicos que trabalhavam em UTI apenas de forma temporária, para fins de complementar sua renda. Inclusive, consistia em um grupo de jovens médicos, em sua maioria recém-formados, com altas cargas de trabalho, o que, não diferente do esperado, ocasiona um desgaste e exaustão mental e corporal, revelando se tratar de perfil de fácil instauração da síndrome.

\section{Considerações Finais}

As Unidades de Tratamento Intensivo (UTI's), consistem em locais que carecem de mais análises e estudos, tendo em vista que têm se apresentado como ambiente desencadeador de estímulos nocivos -físicos e mentais-, que tem dado causa ao desenvolvimento de desequilíbrios psicológicos e orgânicos nos profissionais da medicina atuantes nesses locais. 
O trabalho de um profissional nessas unidades, carece de habilidades diferenciadas, excesso de atenção e um forte controle emocional, para que o profissional seja capaz de gerir as adversidades que surgem. Ademais, as longas jornadas de trabalho e o ritmo acentuado do local, demandam de muita responsabilidade por parte do profissional, não só em relação a qualidade, mas ao quantitativo de tarefas que surgem.

$\mathrm{O}$ ambiente estressante e exaustivo de um bloco intensivista, ensejam o aparecimento de doenças se trazem sintomas como os de tristeza profunda; desinteresse; desconcentração; episódios de raiva e apatia, sentimento de indignação, sensação de incompetência; vontade recorrente de se autocidar.

Nesse diapasão, tem se verificado o desenvolvimento de enfermidades nesses profissionais, não apenas físicas, em razão das extensão jornadas de trabalho e privação de sono e uma devida alimentação; como também psicológicas, devido a todo estresse, medo e demais sentimentos que a rotina desperta nesses médicos.

Verificou-se, dessa forma, que o Transtorno Mental Comum (TMC) e a Síndrome de Burnout, tem se desenvolvido com facilidade no de ambiente de UTI's, tendo em vista se tratar de local em que há diversos fatores ocasionadores de estresse e quadros depressivos, como a presença de pacientes com quadros clínicos graves, inclusive, com alto risco de morte, e uma rotina intensa de trabalho.

Além disso, os médicos acometidos por essas enfermidades, em sua maioria, são jovens, portadores de elevada carga laboral por semana, que trabalham em UTI's de forma temporária e para complementar renda, e que não possuem formação médica específica em Medicina Intensivista, o que possibilita a delineação de um perfil clínico da síndrome.

Sendo assim, desenvolver um trabalho com apreço, qualidade e dedicação é algo de extrema importância. No entanto, a qualidade de vida -garantida por meio de um equilíbrio entre vida profissional e pessoal, parece ser um fator importante para evitar-se os efeitos da rotina da medicina intensivista. Tal situação, evitaria o cansaço extremo, a ausência de relações sociais e interpessoais, bem como instauração de quadros de estresse, ansiedade e irritação, por exemplo, assim como como para evitar que esses profissionais recorram ao uso de drogas ou almejem a morte. Logo, o incentivo a manutenção de uma qualidade de vida por esses profissionais configura uma forma de prevenir o desencadeamento de enfermidades físicas e mentais. 
Dessa forma, resta controversa sobre que, os profissionais da saúde são o grupo laboral mais expostos a insalubridades físicas e mentais, bem como tendentes a desenvolver distúrbios psicológicos; o que revela a necessidade do desenvolvimento de estudos mais elaborados, para fins de ser possível elaborar métodos estratégicos de enfrentamento dessas enfermidades, bem como de prevenção das mesmas.

\section{Referências}

ALCANTARA, L..; SANT'ANNA, J. L..; SOUZA, M. G. N.. Adoecimento e finitude: considerações sobre a abordagem interdisciplinar no Centro de Tratamento Intensivo oncológico. Ciência \& Saúde Coletiva, 2013, 18(9), p. 2507-2514. Disponível em: <http://www.scielo.br/scielo.php?pid=S1413-81232013000900004\&script=sci_abstract >. Acesso em 18/05/2018.

BARBOSA, A. S.. J. Prevalência de Transtornos Mentais Comuns e fatores associados em estudantes de Medicina da UFBA. Graduação em medicina (Monografia). Universidade Federal da Bahia, Faculdade de Medicina da Bahia. 2016. Disponível em: $<$ https://repositorio.ufba.br/ri/bitstream/ri/21360/1/Allana\%20da\%20Silva\%20Junior\%20\%20 Barbosa\%20-\%20Minuta-2.pdf>. Acesso em 18/05/2018.

BENEVIDES-PEREIRA, A. N. T. Transtornos emocionais e a formação em Medicina: um estudo longitudinal. Revista Brasileira de Educação Médica. 2009, Mar, 33 (1), p. 10 - 23. Disponível em: < http://www.scielo.br/pdf/rbem/v33n1/03.pdf >. Acesso em 18/05/2018.

BOOTH, R.; PETRIE, K. Emotional expression and Health Changes: Can we identify biological pathways? In: S. Lepore \& J. Smyth (Orgs.). The writing cure: How expressive writing promotes health and emotional well-being. Washington: American Psychological Association. pp. 157-176, 2002.

CABANA, M. C. F. L.; LUDERMIR, A. B.; SILVA, E. R.; FERREIRA, M. L. L.; PINTO, M. E. R. Transtornos mentais comuns em médicos e seu cotidiano de trabalho. Jornal Brasileiro de Psiquiatria. 2007; 56(1), p. 33-40. Disponível em: < https://doaj.org/article/3e1ee8b43ff647e6950e645f261250ec>. Acesso em 18/05/2018.

CARVAlhO, C. N.; MELhO-FILHO, D .A.; CARVAlHO, J. A. G.; AMORIM, A. C. G. Prevalência e fatores associados aos transtornos mentais comuns em residentes médicos e da área multiprofissional. Jornal Brasileiro de Psiquiatria, 2013, 62(1), p. 38-45. Disponível em: < http://www.ipub.ufrj.br/portal/jbp/62/01/006_JBP_62(1).pdf >. Acesso em 18/05/2018.

EMBRIACO, N., AZOULAY E., BARRAU K., KENTISH N., POCHARD F., LOUNDOU A. High level of burnout in intensivists: prevalence and associated factors. Am J Respir Crit Care 
Med. 2007;175, p. 686-692. Disponível em: https://www.ncbi.nlm.nih.gov/pubmed/17234905 >. Acesso em 18/05/2018.

FIAMONCINI, R. L.; FIAMONTINI R. E. O stress e a fadiga muscular: fatores que afetam a qualidade de vida dos indivíduos. Revista Digital. Buenos Aires, v. 9, n 66, Nov. 2003. Disponível em: < http://www.efdeportes.com/efd66/fadiga.htm>. Acesso em 18/05/2018.

FORD C.V. Emotional distress in internship and residency: a questionnaire study. Psychiatr Med. n. 1, p. 143-150.1983. Disponível em: Disponível em: < http://www.efdeportes.com/efd66/fadiga.htm>. Acesso em 18/05/2018>. Acesso em $18 / 05 / 2018$

GARCIA, E. A. C. O estresse e a saúde ocupacional do médico-intensivista: estudo de casos em uti de Brasília. 2010. Disponível em: < https://s3.amazonaws.com/academia.edu.documents/44851467/estresse_saude_ocupacional.p df?AWSAccessKeyId=AKIAIWOWYYGZ2Y53UL3A\&Expires $=1526716541 \&$ Signature $=1 \mathrm{~s}$ s2cJEOwG\%2FF5Y4mViOW3C26Xuc\%3D\&response-contentdisposition=inline $\% 3 \mathrm{~B} \% 20$ filename\%3DO_ESTRESSE_E_A_SAUDE_OCUPACIONAL_D O_MEDI.pdf $>$. Acesso em 18/05/2018.

GOLDBERG, D. A bio-social model for common mental disorders. Acta Psychiatr Scand Suppl. 1994; 385, 3 p. 66-70. Disponível em: <https://www.ncbi.nlm.nih.gov/pubmed/7740974>. Acesso em 18/05/2018.

GOLDBERG, D.; HUXLEY, P. Common mental disorders: a bio-social model. Primeira edição, ed. London: Tavistock/Routledge; 1992.

GUNTUPALLI K.K.; FROMM JR, R.E. Burnout in the internist-intensivist. Intensive Care Med. 1996; 22, p. 625-630. https://link.springer.com/article/10.1007/BF01709737 >. Acesso em 18/05/2018.

KOVACS, M. J. Sofrimento da equipe de saúde no contexto hospitalar: cuidando do cuidador profissional. O Mundo da Saúde, 34 (4), p. 420-429. 2010. Disponível em: < https://www.saocamilo-sp.br/pdf/mundo_saude/79/420.pdf>. Acesso em 18/05/2018.

LAZARUS, R.; FOLKMAN, S. Stress, appraisal and coping. New York: Springer, 1984.

LOPEZ, A. D.; MATHERS, C. D. A.D.; EZZATI, M., JAMSON, D. T.; MURRAY, C. J. L.., editors; WHO. Global burden of disease and risk factors. New York: Oxford University Press; 2006.

MARI, J. J.; JORGE, M.R.. Transtornos psiquiátricos na clínica geral. Psychiatry On-line Brazil. 1997. Disponível em: < http://www.polbr.med.br/ano97/tpqcm.php >. Acesso em 18/05/2018. 
MASLACH, C.; SCHAUFELI W.B.; LEITER, M. P. Job burnout. Annual Reviews of Psychology. 2001, vol. 52, p. 397-422. Disponível em: < http://www.wilmarschaufeli.nl/publications/Schaufeli/154.pdf>. Acesso em 18/05/2018.

MELEIRO, A.M.A.S. Suicídio entre médicos e estudantes de medicina. Revista da Associação Médica Brasileira, São Paulo, vol.44, n.2, Abril/junho, 1998. Disponível em: <http://www.scielo.br/scielo.php?script=sci_arttext\&pid=S0104-42301998000200012>. Acesso em 18/05/2018.

MONTEIRO, M. C.; MAGALHÃES, A. S.; FÉRES-CARNEIRO, T.; MACHADO, R. N. Terminalidade em UTI: dimensões emocionais e éticas do cuidado médico intensivista. Revista Psicologia em Estudo. 2016; 21(1), p. 65-75. Disponível em: < https://doaj.org/article/4dc7a96f84434b0bb4fd03fa5e06b5f9>. Acesso em 18/05/2018.

PITTA A. Hospital: dor e morte como ofício. São Paulo: HUCITEC; 1994.

SOBRINHO, C. L. N.; BARROS, D. S.; TIRONI, M. O. S.; MARQUES FILHO, E.D S. Médicos de UTI: prevalência da Síndrome de Burnout, características sociodemográficas e condições de trabalho. Revista Brasileira de Educação Médica., 34(1), p. 106-115. Disponível em: < https://doaj.org/article/b51f040b43e44c31974f7023d4f89f92>. Acesso em 18/05/2018.

SPRICIGO, J.S. Saúde Mental e Dependência Química. Universidade Federal de Santa Catarina. Centro de Ciências da Saúde, Florianópólis. 2010, p. 98 Disponível em: < https://unasus.moodle.ufsc.br/file.php/50/PDF/pdf_221010/Modulo9_SaudeMental_un1.pdf >. Acesso em 18/05/2018.

THOMAS, N.K. Resident burnout. JAMA. 2004; 292, p. 2880-2889. Disponível em: < https://jamanetwork.com/journals/jama/fullarticle/199994 >. Acesso em 18/05/2018.

TYSSEN R. Health problems and the use of health services among physicians: a review article with particular emphasis on Norwegian studies. Ind Health. 2007; 45(5), p. 599-610. Disponível em: <https://www.ncbi.nlm.nih.gov/pubmed/18057803>. Acesso em 18/05/2018.

\section{Como citar este artigo (Formato ABNT):}

HOLANDA JÚNIOR, Pedro Henrique de; ALENCAR, Francisca Silva de; NOBRE, Juliane de Oliveira Costa. O Trabalho Médico nas Unidades de Terapia Intensiva (UTI's) e a propensão ao desenvolvimento de Distúrbios Psicológicos . Id on Line Rev.Mult. Psic., 2018, vol.12, n.41, p.39-51. ISSN: 1981-1179.

Recebido: $17 / 05 / 2018$

Aceito 05/06/2018 